\title{
Prospective study comparing ulnar neurolysis in situ with subcutaneous transposition treatment for ulnar impairment in leprosy
}

\begin{abstract}
Introduction: Leprosy patients submitted to surgical decompression and neurolysis of ulnar nerve in the elbow were randomly assigned to simple decompression or transposition of the nerve.

Methods: In order to evaluate the differences in both techniques 136 patients were blindly enrolled into two groups. The patients were evaluated one month, three months and six months after surgery.
\end{abstract}

Results: Pain improved in all patients and there was no difference between the groups. Recovery of sensation and motor performance didn't show significant improvement in both groups.

Conclusion: Simple decompression or transposition of the ulnar nerve are equally efficient in treating ulnar nerve compression in leprosy.

Keywords: leprosy, quality of life, chronic pain, mycobacterium leprae, peripheral nerves

\section{Introduction}

Leprosy was first identified 600 B.C. in China, Egypt, and India. In 1864 Gerhard Armour Hansen made the correlation between the illness and the infectious agent. ${ }^{1}$ The mean time between infection and clinical symptoms is 5 years. ${ }^{1-3}$

There is an infected population of 10 million people in the world distributed predominantly in Africa, Asia and Latin America. The natural immune protection occurs in $95 \%$ of the population. ${ }^{1}$ Leprosy is an endemic disease particularly in Brazil which represents the second most prevalent country in the world supplanted only by India. The peripheral nerve involvement is usually accompanied by physical deformities. $^{4-7}$

Involvement of peripheral nerves in leprosy is characterized by tissue swelling and sometimes replacement by fibrosis. ${ }^{8,9}$ Median, ulnar, posterior tibial and fibular nerves are usually damaged. The low body temperature in these sites provides the best environment for the bacillus. ${ }^{1,10,11}$ The surgical treatment is an option and selected cases. The surgery is delayed for 4 months after no improvement of pain or when observed severe peripheral nerve involvement characterized by sensory loss, or decrease in muscular strength.

Leprosy patients presenting with ulnar compression were submitted to two techniques, simple decompression and subcutaneous transposition, and evaluated on follow up for sensibility, referred pain and muscle strength with the propose of comparing the both the techniques efficacy when considering functional outcomes.

\section{Methods}

Altogether, 215 subjects with compression neuropathy were submitted to 412 surgeries between February 2009 and March 2013 in the Hospital Colonna Dom Rodrigo de Menezes of the Public Health System in Salvador-Bahia. The mean age was 41,06 (8 and 87 years (mean,sd 14,57). Ulnar nerve was the most frequently involved, followed by the posterior tibial median and fibular nerve. We enrolled
Volume 9 Issue 2 - 2019

\author{
José Marcos Pondé Fraga Lima,' Carlos Dias \\ Ribeiro Neto, ${ }^{2}$ Lázaro Lacerda Santos ${ }^{2}$ \\ 'Doctor in neurosurgery, Escola Bahiana de Medicina e Saúde \\ Pública and Universidade Federal da Bahia, Brazil \\ ${ }^{2}$ Medical students of the institution Escola Bahiana de Medicina \\ e Saúde Pública, Brazil
}

Correspondence: José Marcos Pondé Fraga Lima, Doctor in neurosurgery, Escola Bahiana de Medicina e saude publica and Universidade Federal da Bahia, Brazil, Tel +55 7l 98894 - 8934, Email vesalio@terra.com.br

Received: March 04, 2018 | Published: March 28, 2019 a total of 136 patients, of whom 82 patients were males and 54 were females, as can be seen in Figure 1.

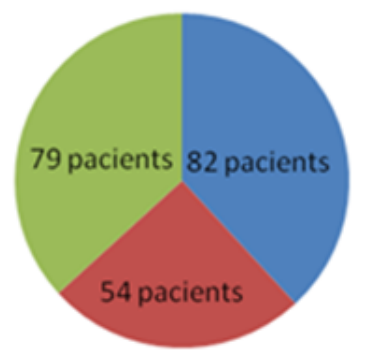

rman

woman

other compressive neuropathy

Figure I Distribution of patients submitted to ulnar neurolysis and decomposition between 2009 and 2013 by gender.

The hospital ethical committee authorized the procedure and the patients were informed about the surgery and its risks, having all of them signed the informed consent term. The right side was involved in 72 patients, the left side in 64 and both in 6 . In these 6 patients we made transposition in one side and simple decompression in the other.

As an open label trial, patients with ulnar compression were alternatively and blindly assigned into two Groups, with 68 patients each, according to the order of admission in the surgical theater. Group I patients were submitted to subcutaneous transposition of the nerve and Group II patients were submitted to simple decompression. In 4 patients due to nerve swelling the transposition was impossible and simple decompression was done. In these situations, if necessary, we subsequently transposed the nerve of two patients in order to follow the randomization pattern.

Pain was the most common complaint and was evaluated with the visual analogical scale (0 to 10$)$. The sensibility was quantified by means of the Siemmens Weinberg monofilaments at all the hand's fingers. The pinprick sensations varied between 0 to 5 . The 0 value is complete loss of sensibility and 5 normal sensibility. A value above 2 is a meaningful evidence of protection against warm or cold injury. 
The motor performance was always quantified with adduction of the thumb. The values varied between M0 and M5 with M3 representing motion against gravity, according to the Medical Research Council muscular strength scale. The patients were followed for a minimal of 6 months and were evaluated for referred pain; motor strength and sensibility at 2 months; 4 months and 6 months after surgery.

\section{Surgery}

Patients submitted to both simple decompression and subcutaneous transposition were put under local anesthesia with lydocaine $2 \%$ and epinephrine without tourniquet and the arm abducted. A curvilinear incision $7 \mathrm{~cm}$ longer is made anterior to the medial epicondyle of the umerus. The subcutaneous vessels are coagulated with bipolar. The dissection of the subcutaneous tissue inside the superficial plan leaves a fasciodermal sling over the fascia of flexor-pronator muscles utilized to cover the ulnar nerve in case of transposition. After retraction of the two lips of the incision, the ulnar nerve is dissected between the medial epicondyle of umerus and olecranus. After section of the ligament between the two bones, the ulnar nerve is totally freed from its natural bed. It is very important to dissect as proximally as possible intending to section the arcade of Struthers and the medial septum points of compression of the ulnar nerve. The distal dissection is carried until the two divisions of the flexor carpi ulnar muscle are reached. The articular branches of the ulnar nerve are sectioned in order to take off the nerve from his natural bed. The motor branch to flexor carpi ulnar muscle is preserved and the channel that subsides after the nerve mobilization is closed with 3-0 nylon. The subcutaneous is closed in one layer in case of single decompression with vycril 2-0 or in two layers in case of transposition involving fasciodermal sling of subcutaneous tissue previously dissected. The skin is closed with simple running suture with mononylon 3-0. A bandage is applied without compression. The skin suture is removed with 3 weeks. No immobilization is required and the surgery takes over 20 minutes with the patients discharged home. Image 1 show a hypertrophied ulnar nerve and Image 2 shows the simple decompression of the ulnar nerve in a patient diagnosed with leprea.

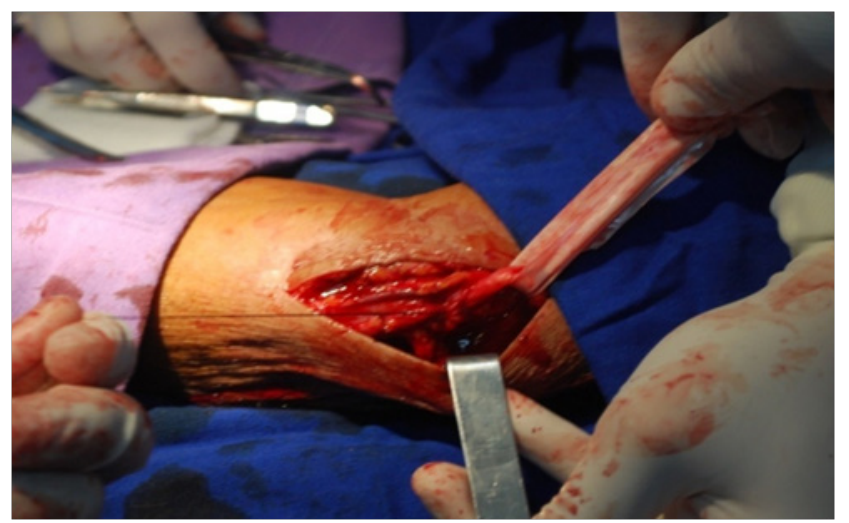

Image I Hypertrophied ulnar nerve.

Statistical analysisThe statistical analysis of the data showed a pattern of normal distribuition and the parameters for referred pain; sensibility and motor strength were evaluated through means of the parametric test of $\mathrm{t}-$ student. The alpha value was equal to 0,05 .

\section{Results}

Time relapse between neurological compromise and surgical treatment varied from 4 to 18 months (mean 8).
Patients that had been submitted to surgery on both arms referred a sense of better functionality in the side submitted to simple decompression when compared with the other transposed one after 6 months of operation.

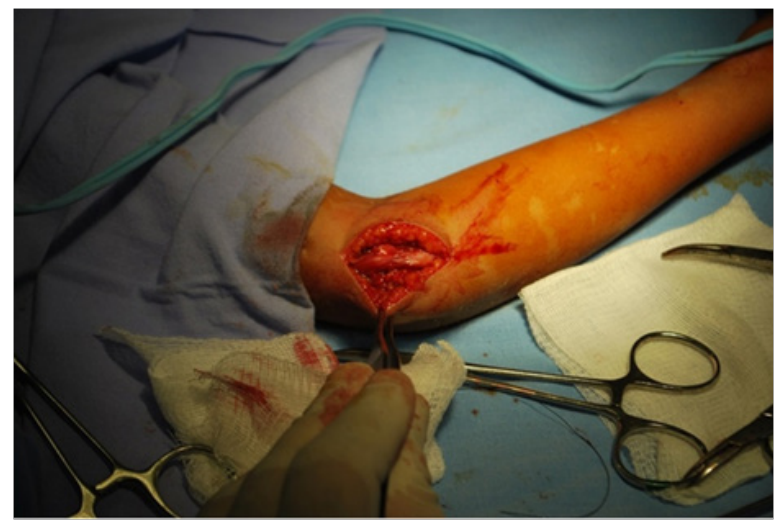

Image $\mathbf{2}$ Simple decompression of the ulnar nerve.

The pain improved in all operated patients. With a mean preoperative referred pain value of 6,96 , group I showed an improvement to a post-operative mean value of 1,93 of referred pain value. With a mean referred pain value of 6,55 , group II showed a decrease to a postoperative mean referred pain value of $1,42(\mathrm{P}<0.0001)$. The sensation increased in both groups. Group I changed from a preoperative value of 1,52 to a mean postoperative value of 2,22.p $>0,05$. Group II changed from a mean preoperative value of 1,31 to a post-operative value of $1,94 \mathrm{p}>0,05$ ( not significant). The muscular strength also improved in both groups although not statistically significant: Group I changed from a preoperative mean value of 2,68 to a mean postoperative value of 2,75.p $>0,05$. Group II changed from a preoperative mean value of 2,52 to a postoperative mean value of 2,64.p> 0,05. There were no statistically significant differences between both groups as $\mathrm{P}>0,05$.

Figure 2 presents the mean change in pain; measured sensitivity and muscle strength for group I after surgery, while Figure 3 presents the mean change for group II after surgery.

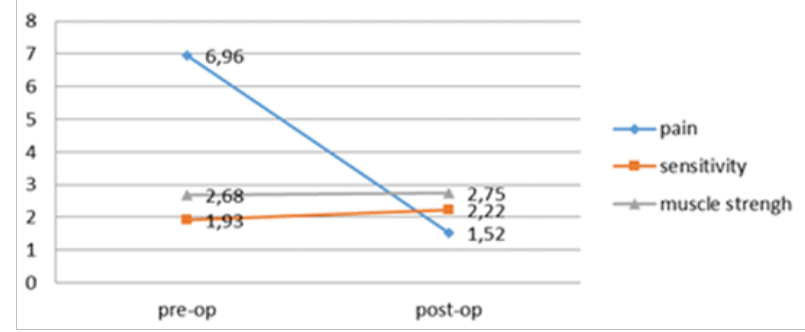

Figure 2 Mean Change in pain; measured sensitivity and muscle strength from pre-operative and post-operative pateints from group I.

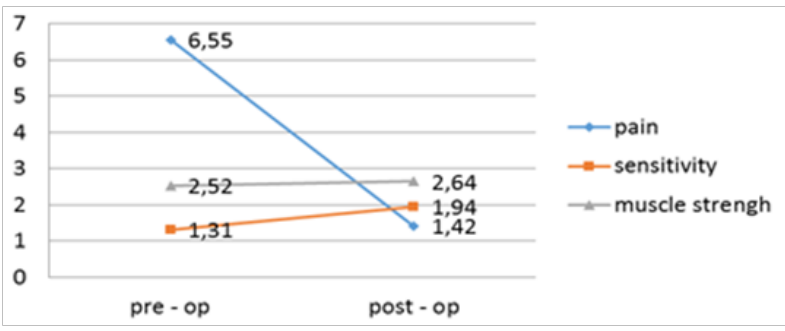

Figure 3 Mean Change in pain; measured sensitivity and muscle strength from pre-operative and post-operative pateints from group II. 


\section{Discussion}

After carpal tunnel syndrome, ulnaropathy at the elbow is the most frequent nerve compression syndrome in the arm. Since its first description in 1816, surgical treatment of ulnar neuropathy at the elbow has received much attention. ${ }^{12}$ The pioneers include Learmonth (submuscular transposition, 1942), King and Morgan (medial epicondylectomy, 1950), Osborne (in situ decompression, 1957), Harrison and Nurrick (subcutaneous transposition, 1970), and Catalano, who in 1980 described the intramuscular placement after transposition. ${ }^{12}$ There are five possible points of compression of the ulnar nerve in the elbow that must be released in leprosy disease in order to decompress the hypertrophied nerve.

The first point of compression is the region of an adherence between the triceps and the medial intermuscular septum called Arcade of Struthers. The second point is the own very medial intermuscular septum. Usually the first and the last points are secondary sites of compression following previous transposition. ${ }^{13}$

The third point lies between the medial epicondyle and the olecranus where the ulnar passes through the cubital tunnel, which cealing is the band of osborne, a facial thickening between the two heads of the flexor carpi ulnar muscle. The fourth point is between the two heads of the flexor carpi ulnar muscle and the last one is in the deep fascia of flexor pronator muscles.

When considering the collective literature of case series and expert opinion, the results were variable and, of course, opposing opinions were found on preferred technique. Justification for recommendations were mainly based on avoiding associated complications of the alternative techniques or an inability to address the perceived pathophysiology adequately. ${ }^{14}$ Biggs and Curtis, ${ }^{14}$ to help clarify the optimal surgical strategy for idiopathic, symptomatic ulnar nerve compression at the elbow, made a prospective study. They recruited forty-four surgical candidates randomized into the neurolysis (n 23) or transposition (n 21) groups. Both procedures were equally effective in producing objective neurological improvement (61\% in the neurolysis group, $67 \%$ in the transposition group). However, complications were more significant in the transposition group. Three of 21 in the transposition group compared with 0 of 23 in the neurolysis group experienced a deep wound infection.

Gervasio et al. ${ }^{15}$ described 70 patients with severe cubital tunnel syndrome: 35 patients were submitted to simple decompression and 35 patients were treated by anterior deep submuscular transposition. No statistically significant difference was found between the two groups with regard to the clinical or the electrophysiological outcome. ${ }^{15}$

Huang et al. ${ }^{16}$ presented the simple decompression technique as the best option in selected cases. Bartels et al. ${ }^{17}$ made a study to compare the costs, from a social standpoint, of simple decompression versus anterior subcutaneous transposition in Euros. The investigators tabulated the actual costs incurred for professional fees, use of the operating room, duration of sick leave, reintervention, and treatment of complications directly related to the surgical treatment. The total median costs per patient were $€ 1124$ for simple decompression and $€ 2730$ for anterior subcutaneous transposition. The main difference was in the costs related to sick leave, which is significantly shorter for simple decompression. There also was a statistically significant difference in operation time in favor of simple decompression. ${ }^{17}$

Transposition is a time consuming procedure when compared with simple decompression although not measured in this paper. There was a trend toward improvement in muscular strength and sensibility after decompression although not statistically significant.

We can explain that the difference as a whole seems not significant because there are a lot of patients operated after 6 months to a year following the beginning of the neurologic damage. Early decompression affords the best results. . $^{5-11}$

\section{Conclusion}

Both simple decompression and subcutaneous transposition have the same results in terms of recovery of sensation, motor strength and pain. Patients submitted to decompression and transposition in opposite arms referred best self-impression in the simple decompressed arm. We can in conclusion recommend simple decompression as treatment to ulnar impairment in leprosy.

\section{Acknowledgments}

None.

\section{Conflicts of interest}

Authors declare no conflicts of interest.

\section{References}

1. Agrawal A, Pandit L, Dalal M, et al. Neurological manifestations of Hansen's disease and their management. Clin Neurol Neurosurg. 2005;107(6):445-454.

2. Croft RP, Nicholls PG, Steyerberg EW, et al. A clinical prediction rule for nerve-function impairment in leprosy patients. Lancet. 2000;355(9215):1603-1606.

3. Naafs B. Bangkok Workshop on Leprosy Research. Treatment of reactions and nerve damage. Int J Lepr Other Mycobact Dis. 1996;64(4 supply):S21-S28.

4. Pandya NJ. Surgical decompression of nerves in leprosy: an attempt at prevention of deformities: A clinical, Electophysiologic, Histophatologic and Surgical study. Int J Lepr Other Mycobact Dis. 1978;46(1):47-55.

5. Husain S, Mishra B, Malaviya GN. Evaluation of results of surgical decompression of median nerve in leprosy in relation to sensory motor function. Acta Leprol. 1997;10(4):199-201.

6. Husain S, Mishra B, Prakash V, et al. Results of surgical decompression of ulnar nerve in leprosy. Acta leprol. 1998;11(1):17-20.

7. Husain S, Mishra B. Decompression of peripheral nerve trunks in leprosy to prevent the development and progression of deformities. Indian J Orthop. 2008;42(1):78-82.

8. Bryceson A, Pfaltzgraff RE. Complications due to nerve damage In Medicine in the tropics: leprosy. 3rd edn, Edinburgh: Churchill Linvigstone. 1990:133-151.

9. Junqueira LCU, Montes CS, Neto EA Barros C, et al. The collagen of permanently damaged nerves in human leprosy. Int $J$ Lepros. 1980;48(3):291-297.

10. Ministry of Health (BR). Department of Primary Health Care. The responsibility of basic care in the diagnosis of leprosy. Basic care report; Brasília: Ministry of Health; 2007.

11. Jain S, Reddy RG, Osmani SN, et al. Childhood leprosy in an urban clinic, Hyderabad, India: clinical presentation and the role of household contacts. Lep Rev. 2002;73(3):248-253.

12. Bartel RH. History of the Surgical Treatment of Ulnar Nerve Compression at the Elbow. Neurosurgery. 2001;49(2):391-400. 
13. Tang P, Plancher KD, Bishai SK. Anterior Submuscular Transposition of the Ulnar Nerve. 2006;21(4):318-324.

14. Biggs M, Curtis JA. Randomized, prospective study comparing ulnar neurolysis in situ with submuscular transposition. Neurosurgery. 2006;58(2):296-304

15. Gervasio O, Gambardella G, Zaccone C, et al. Simple decompression versus anterior submuscular transposition of the ulnar nerve in severe cubital tunnel syndrome: a prospective randomized study. Neurosurgery. 2005;56(1):108-117.
16. Huang JH, Samadani U, Zager EL. Ulnar nerve entrapment neuropathy at the elbow: simple decompression. Neurosurgery. 2004;55(5):11501153 .

17. Bartels RH, Termeer EH, van der Wilt GJ, et al. Simple decompression or anterior subcutaneous transposition for ulnar neuropathy at the elbow: a cost-minimization analysis-part 2. Neurosurgery. 2005;56(3):531-536.

18. VanVeen NHJ, Schreurs TAR, Theuvenet WJ, et al. Decompressive surgery for treating nerve damage in leprosy. A Cochrane review Lepr Re. 2009;80:3-12 